\title{
Operation $\triangle$ as the tool for the de-i-fuzzification procedure and for the correction of the unconscientious experts' evaluations
}

\author{
Lilija Atanassova $^{1}$ and Piotr Dworniczak ${ }^{2}$ \\ ${ }^{1}$ Institute of Information and Communication Technologies, \\ Bulgarian Academy of Sciences \\ Acad. Georgi Bonchev Str., Bl. 2, Sofia-1113, Bulgaria \\ e-mail: 1.c.atanassova@gmail.com \\ ${ }^{2}$ The Great Poland University of Social and Economics \\ ul. Surzyńskich 2, 63-000 Środa Wlkp., Poland \\ e-mail: p.dworniczak@wwsse.pl
}

Received: 16 June 2021

Accepted: 20 November 2021

\begin{abstract}
In this paper, the $\triangle$ operation is discussed as an appropriate tool for the de-i-fuzzification procedure. Some properties of the $\triangle$ operation are presented. A known way of the correction of the unconscientious experts' evaluations in the intuitionistic fuzzy environment is given as the result of the $\triangle$ operation.
\end{abstract}

Keywords: Operation on intuitionistic fuzzy sets, Unconscientious experts' evaluations correction, De-i-fuzzification procedure.

2020 Mathematics Subject Classification: 03B52, 03E72, 68T27, 68T37, 94D05.

\section{Introduction}

In 1983, Krassimir T. Atanassov presented in [5] the concept of some kind of vague sets, called Intuitionistic Fuzzy Sets (IFSs).

Definition 1 ([5]). Let U be a fixed, classical set. An Intuitionistic Fuzzy Set (for shortly: IFS) A over the universe $U$ is defined as

$$
A=\left\{\left\langle x, \mu_{A}(x), v_{A}(x)\right\rangle \mid x \in U\right\},
$$


where $\mu_{A}, v_{A}: U \rightarrow[0,1]$ are the membership degree and non-membership degree of the element $x$ to the set $A$, respectively, and

$$
\mu_{A}(x)+v_{A}(x) \leq 1
$$

for any $x \in U$.

The concept is directly alluded to the concept of Fuzzy Sets (FSs) introduced in 1965 by L.A. Zadeh. IFS, however, differs from FS, because an independence (besides the condition $\mu_{A}(x)+$ $\left.v_{A}(x) \leq 1\right)$ of the membership degree and non-membership degree of element $x$ to the set $A$ was introduced. While in the FS the non-membership degree of an element $x$ to the FS $A$ is equal to $1-\mu_{A}(x)$, where $\mu_{A}(x)$ is the membership degree, Atanassov introduced separate values $\mu_{A}(x)$ and $v_{A}(x)$ of memberships and non-memberships of $x$ to the IFS $A$.

For such a set we can define for any $x \in U$ the value

$$
\pi_{A}(x)=1-\mu_{A}(x)-v_{A}(x)
$$

called the hesitancy degree (degree of indeterminacy) of the element $x$.

Three special cases of IFSs are the sets $E^{*}, F^{*}$, and $F I^{*}$, called the Empty Intuitionistic Fuzzy Set, the Full Intuitionistic Fuzzy Set and the Full Ignorance Intuitionistic Fuzzy Set, defined as follows:

$$
\begin{gathered}
E^{*}=\{\langle x, 0,1\rangle \mid x \in U\}, \\
F^{*}=\{\langle x, 1,0\rangle \mid x \in U\}, \\
F I^{*}=\{\langle x, 0,0\rangle \mid x \in U\} .
\end{gathered}
$$

The sets can be named also complete falsity set, complete truth set and complete uncertainty set, respectively (see: [11]).

For these sets the hesitancy degree are for any element $x \in U$ equal to 0,0 , and 1 , respectively.

In the Intuitionistic Fuzzy Logic (IFL) the truth-value of a variable $x$ is given by ordered pair $\langle a, b\rangle$, where $a, b, a+b \in[0,1]$. Such pair we will call (see: [9]) an Intuitionistic Fuzzy Pair (IFP). The numbers $a$ and $b$ are interpreted as the degrees of validity and non-validity of $x$. We denote the truth-value of $x$ by $V(x)$.

The variable with truth-value True in the classical logic we denote by $\underline{1}$ and the variable False by $\underline{0}$. Therefore, for these variables hold: $V(\underline{1})=\langle 1,0\rangle$ and $V(\underline{0})=\langle 0,1\rangle$. We will use also the

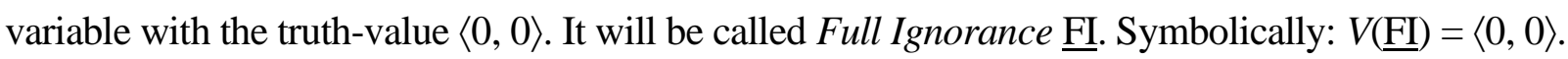
Such a variable does not exist in both the classical and fuzzy logic.

We call the variable $x$ an Intuitionistic Fuzzy Tautology (shortly: IFT), if and only if for $V(x)=\langle a, b\rangle$ holds: $a \geq b$ and, similarly, an Intuitionistic Fuzzy co-Tautology (IFcT), if the inequality $a \leq b$ holds.

The IFP can be also considered in isolation from IFL only as the pair $\langle a, b\rangle$ of numbers, where $a, b, a+b \in[0,1]$.

For the IF pairs we can defined different operations. One of them is introduced in [10] and considered later in [11]. 
Definition 2 ([10]). For IFPs $\langle a, b\rangle$ and $\langle c, d\rangle$, where $a+b+c+d\rangle 0$, the $\triangle$ operation is defined as follows:

$$
\langle a, b\rangle \triangle\langle c, d\rangle=\left\langle\frac{a+c}{a+b+c+d}, \frac{b+d}{a+b+c+d}\right\rangle .
$$

Additionally, we assume that

$$
\langle 0,0\rangle \triangle\langle 0,0\rangle=\langle 0.5,0.5\rangle
$$

Let us notice that in the paper [10] the special value $\langle 0,0\rangle \triangle\langle 0,0\rangle$ is assumed to be equal to $\langle 0,0\rangle$. It is really a rare computational case, but there exist some serious reasons to correct this assumption with $\langle 0,0\rangle \triangle\langle 0,0\rangle=\langle 0.5,0.5\rangle$ as in [11].

Remark 1 ([10]). Directly from the definition it follows that the $\triangle$ operation is commutative, i.e.,

$$
\langle a, b\rangle \triangle\langle c, d\rangle=\langle c, d\rangle \triangle\langle a, b\rangle .
$$

Remark 2 ([10]). For any IFPs $\langle a, b\rangle$ and $\langle c, d\rangle$

$$
\frac{a+c}{a+b+c+d}+\frac{b+d}{a+b+c+d}=1 \text {. }
$$

The operation $\triangle$ can be also designated for the IFSs. In this case, it is defined as follows.

Definition 3 ([10]). For IFSs $A=\left\{\left\langle x, \mu_{A}(x), v_{A}(x)\right\rangle \mid x \in U\right\}$ and $B=\left\{\left\langle x, \mu_{B}(x), v_{B}(x)\right\rangle \mid x \in U\right\}$, such that for each $x \in U$ :

$$
\mu_{A}(x)+v_{A}(x)+\mu_{B}(x)+v_{B}(x)>0
$$

the $\triangle$ operation is defined as follows:

$$
A \triangle B=\left\{\left\langle x, \frac{\mu_{A}(x)+\mu_{B}(x)}{\mu_{A}(x)+v_{A}(x)+\mu_{B}(x)+v_{B}(x)}, \frac{v_{A}(x)+v_{B}(x)}{\mu_{A}(x)+v_{A}(x)+\mu_{B}(x)+v_{B}(x)}\right\rangle \mid x \in U\right\} .
$$

If for some $y \in U$ there is $\mu_{A}(y)+v_{A}(y)+\mu_{B}(y)+v_{B}(y)=0$, then we assume

$$
\left\langle y, \frac{\mu_{A}(y)+\mu_{B}(y)}{\mu_{A}(y)+v_{A}(y)+\mu_{B}(y)+v_{B}(y)}, \frac{v_{A}(y)+v_{B}(y)}{\mu_{A}(y)+v_{A}(y)+\mu_{B}(y)+v_{B}(y)}\right\rangle=\langle y, 0.5,0.5\rangle .
$$

Numerical example 1. Let $U=\{x, y, z, t\}$ and the IFSs $A$ and $B$ are:

$$
\begin{aligned}
& A=\{\langle x, 0.5,0.5\rangle,\langle y, 0.7,0.0\rangle,\langle z, 0.1,0.4\rangle,\langle t, 0.6,0.2\rangle\}, \\
& B=\{\langle x, 0.3,0.7\rangle,\langle y, 0.0,0.0\rangle,\langle z, 0.3,0.2\rangle,\langle t, 0.4,0.4\rangle\},
\end{aligned}
$$

then

$$
A \triangle B=\{\langle x, 0.4,0.6\rangle,\langle y, 1.0,0.0\rangle,\langle z, 0.4,0.6\rangle,\langle t, 0.625,0.375\rangle\}
$$

\section{Numerical example 2. For}

$$
\begin{aligned}
& E^{*}=\{\langle x, 0,1\rangle \mid x \in U\}, \\
& F^{*}=\{\langle x, 1,0\rangle \mid x \in U\}, \\
& F I^{*}=\{\langle x, 0,0\rangle \mid x \in U\},
\end{aligned}
$$

we have 


$$
\begin{aligned}
E^{*} \triangle E^{*} & =E^{*}, \\
E^{*} \triangle F^{*} & =\{\langle x, 0.5,0.5\rangle \mid x \in U\}, \\
E^{*} \triangle F I^{*} & =E^{*}, \\
F^{*} \triangle F^{*} & =F^{*}, \\
F^{*} \triangle F I^{*} & =F^{*}, \\
F I^{*} \triangle F I^{*} & =\{\langle x, 0.5,0.5\rangle \mid x \in U\} .
\end{aligned}
$$

Remark 3. For IFSs $A$ and $B$ on the universe $U$ the set $A \triangle B$ is a fuzzy set on the universe $U$.

In $[10,11]$ the geometrical interpretation of the $\triangle$ operation is presented.

Let us notice, that formally there exists a conflict of notation in Definition 2 and Definition 3. In the first place, the sign $\triangle$ denotes the operation between IF pairs while in the Definition 3 the operation between IF sets. Nevertheless, we will use the same sign $\triangle$ in both cases.

In the next sections the operation will be used for the procedure of de-i-fuzzification of the IFSs and for the correction of the unconscientious experts'evaluations.

\section{Operation $\triangle$ and the de-i-fuzzification procedure}

De-i-fuzzification procedures have been under development since 1988. First, remarks on this subject are presented by Atanassov. He noticed in [6] that the then introduced operator $D_{\alpha}$ transforms the IF set into an ordinary fuzzy set. In this paper, the term de-i-fuzzification is not formally mentioned.

Presumably, the next paper in this direction is [1]. Angelov calls the presented procedure a crispification. In his paper, the crispification is the mapping, linking the IFS $A$ on the universe $U=\Re$ with a real number. Angelov presents the crispification as a procedure analogous to the well-known defuzzification type COG/COA (center of gravity / center of area), MOM (mean of maxima) or BADD (basic defuzzification distributions) presented earlier in [17, 18], for the classical fuzzy sets.

Because the result of the $\triangle$ operation on the IFS $A$ is an ordinary fuzzy set, the operation can be used for the procedure of de-i-fuzzification. The procedure of de-i-fuzzification allows to transform the IFSs (proper IFSs) into a (ordinary) fuzzy set. The procedure can be important especially for the application of the IFSs in the case when the result as FSs or a real number is required. As we already mentioned, the consideration about the changing IFSs into FSs or real numbers are presented in 1988 in [6], and developed later in [1-4, 12, 13, 19-21]. We should note that the literature about this subject is not abundant, although, there exist several procedures of conversion of IFSs into FSs or real numbers.

The crispification, de-i-fuzzification and defuzzification can be illustrated as follows on Fig. 1 . 


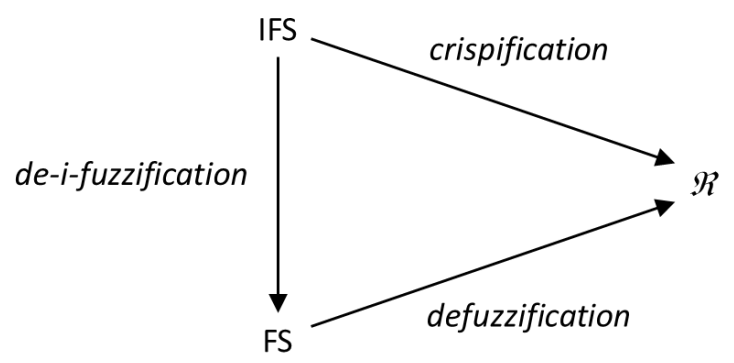

Figure 1. The crispification, de-i-fuzzification and defuzzification mappings

The proper de-i-fuzzification procedure maps the IFS $A$ in the FS $A^{\prime}$. It is therefore the mapping $D$ : $\operatorname{IFS}(U) \rightarrow \operatorname{FS}(U)$, where $\operatorname{IFS}(U)$ is the family of the IFSs on the universe $U$ and $\mathrm{FS}(U)$ is the family of the FSs on the universe $U$. The literature on de-i-fuzzification (or crispification) is quite scarce, although the application of IFSs to the technique would require case-by-case development as well as general research. In the existing literature on the subject no general properties of such procedures are given (see: $[1-4,12,13,19-21]$ ).

In basic monographs on the IFSs theory such conditions also do not exist (see: [7, 8]).

In a special case, the de-i-fuzzification procedure can be considered as some projection of the point $\langle a, b\rangle$, where $a+b \leq 1$, on a straight line $a+b=1$. The result of the projection should be therefore a point $\left\langle a^{\prime}, b^{\prime}\right\rangle$ for which $a^{\prime}+b^{\prime}=1$. If we take the central projection with the center of projection to be the point $O$ with coordinates $\langle 0,0\rangle$, then the result of the projection of the points $\langle a, b\rangle \neq\langle 0,0\rangle$ of the IF interpretational triangle, is a point with coordinates

$$
\left\langle a^{\prime}, b^{\prime}\right\rangle=\left\langle\frac{a}{a+b}, \frac{b}{a+b}\right\rangle .
$$

The result can be obtained by simple using the elementary analytical geometry.

We assume additionally, the "central projection" of the $\langle 0,0\rangle$ is equal to $\langle 0.5,0.5\rangle$.

For the given point $\langle a, b\rangle$, the point $\left\langle\frac{a}{a+b}, \frac{b}{a+b}\right\rangle$ is the only point preserving of the ratio (proportion) of $a$ to $b$. It means, for the given point $\langle a, b\rangle$ if $\frac{a^{\prime}}{b^{\prime}}=\frac{a}{b}$ and $a^{\prime}+b^{\prime}=1$, then $\left\langle a^{\prime}, b^{\prime}\right\rangle=\left\langle\frac{a}{a+b}, \frac{b}{a+b}\right\rangle$. Of course, for $b=0$ and $a \neq 0$ the ratio $\frac{a}{b}$ has no sense and we take $\left\langle a^{\prime}, b^{\prime}\right\rangle=\langle 1,0\rangle$. Similarly, for $b=0$ and $a=0$ the ratio has no sense and we take $\left\langle a^{\prime}, b^{\prime}\right\rangle=\langle 0.5,0.5\rangle$.

The property of keeping the proportions can be considered as one of the most important properties of the de-i-fuzzification procedure.

Let us marginally note that if we take some "infinitely far" center of projection, it is if the center of projection would be $\langle-\infty,-\infty\rangle$, then we come to the orthogonal projection, used in fact in de-i-fuzzification procedure as well (see [13]).

After these considerations we can formulate some remarks. 
Remark 1. The value $\langle a, b\rangle \triangle\langle a, b\rangle=\left\langle\frac{a}{a+b}, \frac{b}{a+b}\right\rangle$ is the fuzzy image of the intuitionistic fuzzy pair $\langle a, b\rangle$ in the central projection with the center $\langle 0,0\rangle$.

Remark 2. For any IFP $\langle a, b\rangle$ the equality:

$$
\langle a, b\rangle \triangle\langle a, b\rangle=\langle a, b\rangle \triangle\langle 0,0\rangle=\left\langle\frac{a}{a+b}, \frac{b}{a+b}\right\rangle
$$

holds.

Remark 3. For the given point $\langle a, b\rangle$ there exist infinitely many points with the projection image in the form of $\left\langle\frac{a}{a+b}, \frac{b}{a+b}\right\rangle$.

Remark 4. If the IFP $\langle a, b\rangle$ is an IFT, then $\left\langle\frac{a}{a+b}, \frac{b}{a+b}\right\rangle$ is an IFT, and if the IFP $\langle a, b\rangle$ is an $\mathrm{IFcT}$, then $\left\langle\frac{a}{a+b}, \frac{b}{a+b}\right\rangle$ is an IFcT.

The central projection is illustrated on Fig. 2.

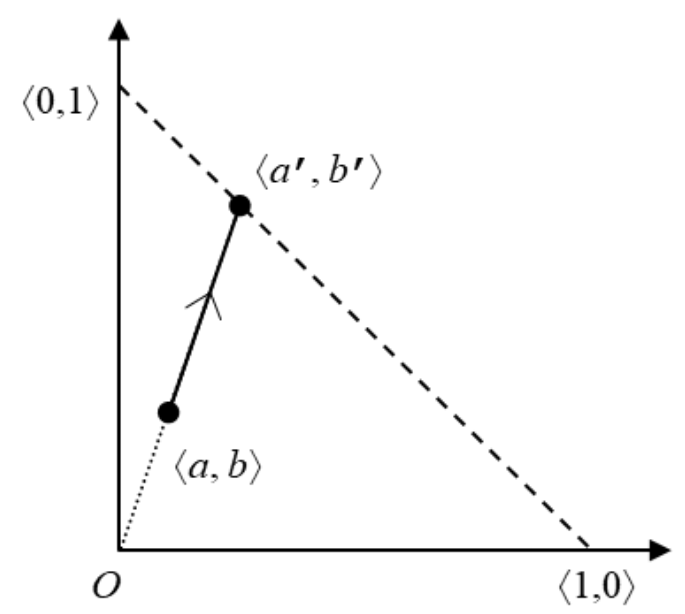

Figure 2. The central projection of the point $\langle a, b\rangle$ of the IF interpretational triangle

The same projection can be considered not only for IFPs but for the whole IF sets.

Theorem 1. For IFSs $A=\left\{\left\langle x, \mu_{A}(x), v_{A}(x)\right\rangle \mid x \in U\right\}$, the set

$$
D I F(A)=A \triangle A=A \triangle F I^{*}=\left\{\left\langle x, \frac{\mu_{A}(x)}{\mu_{A}(x)+v_{A}(x)}, \frac{v_{A}(x)}{\mu_{A}(x)+v_{A}(x)}\right\rangle \mid x \in U\right\}
$$

is the FS preserving the ratio of membership and non-membership function values of the IFS A.

Proof: Follows from the definition of the operation $\triangle$. 
Remark 5. The fuzzy set is typically described only by membership function $\mu$. The nonmembership function is always equal to $1-\mu$. The membership function $\mu_{D I F}: D I F(A) \rightarrow \Re$, of the fuzzy set $\operatorname{DIF}(A)$ is based on the $\mu_{A}$ and $v_{A}$ functions and for any $x \in U$ it is

$$
\mu_{D I F}(x)=\frac{\mu_{A}(x)}{\mu_{A}(x)+v_{A}(x)} .
$$

If $\mu_{A}(x)=v_{A}(x)=0$ for some $x \in U$, then we assume $\mu_{D I F}(x)=0.5$.

Remark 6. The FS DIF $(A)$ can be called the result of the de-i-fuzzification procedure of the IFS A.

Remark 7. If for the IFP $\langle a, b\rangle$ with $a+b=1$ it is valid:

$$
\langle a, b\rangle \triangle\langle a, b\rangle=\langle a, b\rangle \triangle\langle 0,0\rangle=\langle a, b\rangle .
$$

Remark 8. For the FS $A$ it is $\operatorname{DIF}(A)=A$.

The operation $\triangle$ can be therefore used as a tool in the de-i-fuzzification procedure, thus, the result can be a simple contribution to the solution of Open Problem 23 from [8].

Numerical example 3. Let $U=\{x, y, z, t\}$ and the IFS $A$ is:

$$
A=\{\langle x, 0.5,0.5\rangle,\langle y, 0.7,0.0\rangle,\langle z, 0.1,0.4\rangle,\langle t, 0.6,0.2\rangle\}
$$

then

$$
\operatorname{DIF}(A)=\{\langle x, 0.5,0.5\rangle,\langle y, 1.0,0.0\rangle,\langle z, 0.2,0.8\rangle,\langle t, 0.75,0.25\rangle\}
$$

\section{Operation $\triangle$ and the correction of the unconscientious experts' evaluations}

In the monograph [8], in one of the subsections, the issues regarding the use of experts'opinions to determination of the membership degree and the non-membership degree, with which the evaluated variant belongs/does not belong to the IF set of variants satisfying certain criterion, are discussed. The issue was also studied later in [14-16].

In the IF environment, the experts'evaluation can be considered as an IFP. In this case, some problems can arise if an expert is more than $100 \%$ sure that the variant belongs either to the specific set or to the complement of this set. More precisely, we can describe this fact in terms of membership, and non-membership degrees as follows: we will call the experts' evaluation $\langle a, b\rangle$ an unconscientious evaluation (UE), if $a, b \in[0,1]$ and $a+b>1$.

To apply the intuitionistic fuzzy sets theory to the processing of evaluations, the UE $\langle a, b\rangle$ must be adjusted (converted) to the correct IF pair $\left\langle a^{\prime}, b^{\prime}\right\rangle$ for which $a^{\prime}, b^{\prime} \in[0,1]$ and $a^{\prime}+b^{\prime} \in[0,1]$. The IFP has the hesitation margin equal to $\pi^{\prime}=1-a^{\prime}-b^{\prime}$.

Atanassov [8] notes that the fact of existence of this kind of problems by the evaluation of events distinguishes the decision aid in the intuitionistic fuzzy environment from the decision aid in the (classical) fuzzy environment, where such unconscientious evaluations do not exist (or are very easy to be corrected). 
In the literature (except for [14-16]), no general condition has been imposed that should be fulfilled in order for the conversion to be a proper one. However, we think that the conversion should fulfill at least the properties (P1, P2, P3) given below.

Property 1 (Dworniczak, [14]).

a) If $a \geq b$, then $a^{\prime} \geq b^{\prime}$,

b) If $a \leq b$, then $a^{\prime} \leq b^{\prime}$.

This property can be, equivalently, written in the other notation.

\section{Property 1'.}

a) If the IFP $\langle a, b\rangle$ is an IFT, then $\left\langle a^{\prime}, b^{\prime}\right\rangle$ is an IFT,

b) If the IFP $\langle a, b\rangle$ is an IFcT, then $\left\langle a^{\prime}, b^{\prime}\right\rangle$ is an IFcT.

In the case of unconscientious experts evaluations the sum $a+b$ is too large and must be reduced. The reduction can be done in three ways:

a) we reduce both of the degrees $a$ and $b$ to $a^{\prime}$ and $b^{\prime}$, respectively,

b) we reduce the membership degree $a$ to $a^{\prime}$ leaving the non-membership degree $b^{\prime}=b$,

c) we reduce the non-membership degree $b$ to $b^{\prime}$ leaving the membership degree $a^{\prime}=a$, so as to obtain the corrected IFP $\left\langle a^{\prime}, b^{\prime}\right\rangle$ such that $a^{\prime}+b^{\prime} \leq 1$.

Based on above reasoning the conversion's mapping should fulfill, in addition to the Property 1 also the Property 2.

Property 2 (Dworniczak, [16]).
a) $a^{\prime} \leq a$,
b) $b^{\prime} \leq b$.

This property specifies that we should not increase any of the $a$ and $b$ values.

The last of the proposed properties says that the uncertainty of the assessment should not increase during the correction process.

Property 3 (Dworniczak, [16]). If $\langle a, b\rangle$ is an UE, then, for the corrected value $\left\langle a^{\prime}, b^{\prime}\right\rangle$ the inequality $\pi^{\prime} \leq \pi^{0}$ should be satisfied, where $\pi^{0}=a+b-1$ is called the hesitance degree of the $U E$.

In the previously literature of subject some ways for the adjustment of the values in the unconscientious experts case are proposed. One of the ways is presented in [7, 28]. This way of correction can be viewed in the distance sense by using the so-called SNCF distance (called also French metro metric, radial metric hedgehog metric or other). This type of approach is discussed in [16]. This type of correction of the $\mathrm{UE}\langle a, b\rangle$ leads to the result

$$
\left\langle a^{\prime}, b^{\prime}\right\rangle=\left\langle\frac{a}{a+b}, \frac{b}{a+b}\right\rangle .
$$

For the given $\mathrm{UE}\langle a, b\rangle$, the point $\left\langle\frac{a}{a+b}, \frac{b}{a+b}\right\rangle$ is the closest point in the SNCF metric for 
which the sum of coordinates is less or equal to 1 . It is therefore the closest point which can be considered as correction of the UE $\langle a, b\rangle$. For this kind of correction the Properties 1, 2 and 3 are fulfilled.

This way of UE correction can be obtained also by use of some enlargement of the $\triangle$ operation. In the Definition 2, the operation $\triangle$ is defined for $a, b \in[0,1], a+b \leq 1$. We can use the same calculation for $a, b \in[0,1], a+b>1$.

Definition 4. For pairs $\langle a, b\rangle$ and $\langle a, d\rangle$, where $a, b, c, d \in[0,1]$, the $\triangle^{\prime}$ operation is defined as follows:

Additionally, we assume that

$$
\langle a, b\rangle \triangle^{\prime}\langle c, d\rangle=\left\langle\frac{a+c}{a+b+c+d}, \frac{b+d}{a+b+c+d}\right\rangle .
$$

$$
\langle 0,0\rangle \triangle^{\prime}\langle 0,0\rangle=\langle 0.5,0.5\rangle
$$

Of course, if the pairs $\langle a, b\rangle$ and $\langle c, d\rangle$ are IFPs then $\Delta^{\prime}$ is equal to the operation $\triangle$ from the Definition 2.

Now we can formulate the theorem on the correction of the unconscientious experts' evaluation related to the above operation.

Theorem 2. For any UE $\langle a, b\rangle$ the corrected evaluation $\left\langle a^{\prime}, b^{\prime}\right\rangle=\left\langle\frac{a}{a+b}, \frac{b}{a+b}\right\rangle$ can be calculated by $\triangle^{\prime}$ operation as:

$$
\left\langle a^{\prime}, b^{\prime}\right\rangle=\langle a, b\rangle{\triangle^{\prime}}^{\prime}\langle a, b\rangle=\langle a, b\rangle{\triangle^{\prime}}^{\prime}\langle 0,0\rangle=\left\langle\frac{a}{a+b}, \frac{b}{a+b}\right\rangle .
$$

Proof: By definition of $\triangle^{\prime}$ operation.

The theorem presents in fact the constructive way for the obtaining the correction of the unconscientious experts'evaluation.

\section{Numerical example 4.}

a) For the UE $\langle a, b\rangle=\langle 0.9,0.6\rangle$ the corrected value is $\left\langle a^{\prime}, b^{\prime}\right\rangle=\langle 0.6,0.4\rangle$,

b) For the UE $\langle a, b\rangle=\langle 0.6,0.6\rangle$ the corrected value is $\left\langle a^{\prime}, b^{\prime}\right\rangle=\langle 0.5,0.5\rangle$.

\section{Conclusion}

In the paper, the $\triangle$ operation is presented and used as the tool for the de-i-fuzzification procedure. The simple extension of the $\triangle$ operation is used as the tool for the correction of the unconscientious experts' evaluations. The constructive theorems for these operations are formulated. Some remarks pointing to the usefulness of these proposal are given. Some properties of the $\triangle$ operation, related to the de-i-fuzzification procedure and to the correction of the unconscientious experts'evaluations are presented. The further research can be concerned on the dependencies between the $\triangle$ operation and the other operations defined on intuitionistic fuzzy sets. 


\section{References}

[1] Angelov, P. (1995). Crispification: defuzzification of intuitionistic fuzzy sets. BUSEFAL, $64,51-55$.

[2] Angelov, P. (2001). Multiobjective optimisation in air-conditioning systems: comfort / discomfort definition by IF sets. Notes on Intuitionistic Fuzzy Sets, 7(1), 10-21.

[3] Ansari, A. Q., Siddiqui, S. A., \& Alvi, J. A. (2004). Mathematical techniques to convert intuitionistic fuzzy sets into fuzzy sets. Notes on Intuitionistic Fuzzy Sets, 10(1), 13-17.

[4] Anzilli, L., \& Facchinetti, G. (2016). A new proposal of defuzzification of intuitionistic fuzzy quantities. In: Novel developments in uncertainty representation and processing, Atanassov, K.T., Castillo, O., Kacprzyk, J., Krawczak, M., Melin, P., Sotirov, S., Sotirova, E., Szmidt, E., De Tré, G., Zadrożny, S., (eds.), Springer, Cham, Switzerland, 185-195.

[5] Atanassov, K. T. (1983). Intuitionistic fuzzy sets. VII ITKR Sci. Session, Sofia, (June 1983) (Deposed in Central Sci. - Techn. Library of Bulg. Acad. of Sci., 1697/84) (in Bulgarian). Reprint and English version in: International Journal Bioautomation, 20(Supl. 1), 2016, $1-6$.

[6] Atanassov, K. T. (1988). Review and new results on intuitionistic fuzzy sets. Mathematical Foundations of Artificial Intelligence Seminar, Sofia, 1988, Preprint IM-MFAIS-1-88. Reprinted in: International Journal Bioautomation, 20(Supl. 1), 2016, 7-16.

[7] Atanassov, K. T. (1999). Intuitionistic Fuzzy Sets: Theory and Applications. Springer, Heidelberg.

[8] Atanassov, K. T. (2012). On Intuitionistic Fuzzy Sets Theory. Springer, Berlin.

[9] Atanassov, K. T., Szmidt, E., \& Kacprzyk, J. (2013). On intuitionistic fuzzy pairs. Notes on Intuitionistic Fuzzy Sets, 19(3), 1-13.

[10] Atanassova, L. (2020). A new operator over intuitionistic fuzzy sets. Notes on Intuitionistic Fuzzy Sets, 24(1), 23-28.

[11] Atanassova, L., \& Dworniczak, P. (2021). On the operation $\triangle$ over intuitionistic fuzzy sets. Mathematics, 9(13), Article No. 1518.

[12] Atanassova, V., \& Sotirov, S. (2012). A new formula for de-i-fuzzification of intuitionistic fuzzy sets. Notes on Intuitionistic Fuzzy Sets, 18(3), 49-51.

[13] Ban, A., Kacprzyk, J., \& Atanassov, K. T. (2008). On de-i-fuzzification of intuitionistic fuzzy sets. Comptes rendus de l'Academie bulgare des Sciences, 61(12), 1535-1540.

[14] Dworniczak, P. (2012). A note on the unconscientious experts' evaluations in the intuitionistic fuzzy environment. Notes on Intuitionistic Fuzzy Sets, 18(3), 23-29.

[15] Dworniczak, P. (2013). Further remarks about the unconscientious experts' evaluations in the intuitionistic fuzzy environment. Notes on Intuitionistic Fuzzy Sets, 9(1), 27-31. 
[16] Dworniczak, P. (2015). A distance based correction of the unconscientious experts' evaluations. Notes on Intuitionistic Fuzzy Sets, 21(3), 5-17.

[17] Filev, D. P., \& Yager, R. R. (1991). A generalized defuzzification method via BAD distributions. International Journal of Intelligent Systems, 6, 687-697.

[18] Klir, G., \& Yuan, B., (1995). Fuzzy Sets and Fuzzy Logic, Prentice Hall, New Jersey.

[19] Radhika, C., \& Parvathi, R. (2016). Defuzzification of intuitionistic fuzzy sets. Notes on Intuitionistic Fuzzy Sets, 22(5), 19-26.

[20] Szmidt, E., \& Kacprzyk, J. (1998). A fuzzy set corresponding to an intuitionistic fuzzy set. International Journal of Uncertainty, Fuzziness and Knowledge-Based Systems, 6(5), $427-435$.

[21] Yager, R. R. (2009). Some aspects of intuitionistic fuzzy sets. Fuzzy Optimization and Decision Making, 8, 67-90. 